\title{
Nature nano talks
}

We have launched a series of webinars to engage our audience in a way that is complementary to the publication of articles.

\section{"N} ature Nanotechnology is an interdisciplinary journal that publishes papers of the highest quality and significance in all areas of nanoscience and nanotechnology." This is the statement that can be read in the first lines of the 'Aims \& Scope' of our journal (https://www.nature.com/nnano/about/ aims). As editors, we believe strongly in that statement and we do our best, when handling manuscripts, to satisfy it.

We also feel that to be amongst the leading journals in the field we must engage our community beyond the publication of high-quality research papers. The journal publishes comments and other opinion articles that look at research from different points of view. Each editor also attends conferences and visits research departments to interact directly with scientists.

To increase our engagement, we have now launched a series of webinars that we named Nature Nano Talks. The scope of these events is to provide an informal but professional venue for our community to discuss a range of topics in nanoscience and nanotechnology directly with experts.

It is undeniable that the COVID-19 pandemic has something to do with this initiative. On the one hand, the difficulty in meeting members of our community face to face stimulated us to search different routes. On the other hand, it was exactly the science behind the virus that inspired the first online event that our senior editor Christine Horejs (now at Nature Reviews Materials) organized well before we were thinking of a series. Christine invited Nicole Steinmetz and Ronit Satchi-Fainaro, both experts in nanomedicine, to discuss how in their view nanotechnology can contribute to COVID-19 research (https://go.nature. com/3ssvikE). The discussion touched upon some significant issues. For example, how cold-chain requirements could be addressed to ensure that the vaccines can reach people in underdeveloped regions, who have no access to freezers or fridges. Also, how nanotechnology can provide platforms that can be easily adapted when new infectious diseases spread, a strategy that may have been neglected in funding cycles in the past.

Following up from the success of the first event, our senior editor Chiara Pastore organized a panel discussion with Betty Kim, Michael Mitchell, Ankur Singh and

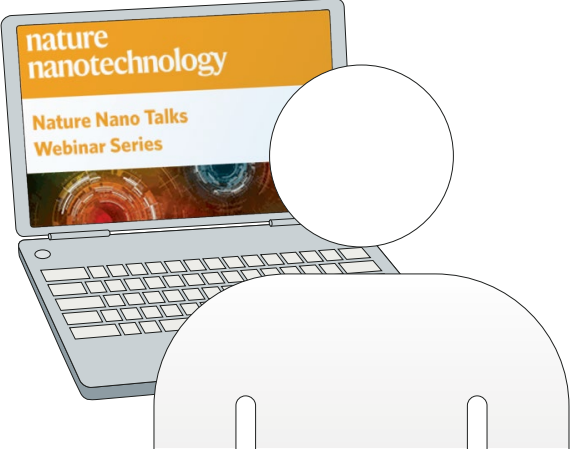

be linked to the presence of polyethylene glycol (PEG) in the nanoparticle composition. In reality, PEG has been approved by the FDA and is already present in other drugs. Effectively, the observed reactions are not higher in number than those observed with other medicines.

Nature Nanotechnology does not only cover nanomedicine and we certainly want to explore other topics in our webinars. In one of the upcoming events, senior editor Wenjie Sun will be talking to experts in nanocatalysis to discuss challenges and opportunities for industrial applications of emerging catalysts (https://

Angus Johnston, all authors of the articles figuring in our Focus issue on nanomaterials for immunomodulation, in particular for cancer immunotherapy and vaccine design (https://go.nature.com/3qW68dQ). As it became clear in the discussion, because of their size nanomaterials can reach specific organs and cells, which is crucial to trigger specific immune responses. For example, nanovaccines with an antigenic cargo can easily travel to the lymph nodes, a key organ system for antibody production, and, in the presence of glycosylated moieties engineered on their surface, specifically target the B cells. Nanotechnology platforms were also highlighted for their potential in delivering multiple therapeutic agents. Namely, cancer vaccines developed with nanomedicine platforms could be designed to present a cocktail of cancer antigens to the immune system, thus broadening the immune response against heterogeneous tumours.

Finally, Chiara and Christine organized a joint Nature Nanotechnology and Nature Reviews Materials Q\&A on lipid nanoparticles for messenger RNA vaccines with Kathryn Whitehead and Yizhou Dong (https://go.nature.com/3aUUZUY). The Q\&A was conceived to explain the technology behind the COVID-19 vaccines and in part also to dispel some of the misconceptions around it. What emerged quite clearly is that despite common belief, lipid nanoparticles for mRNA delivery are not novel. They have been studied and optimized for a long time, and although the leap to the clinic has been fast tracked, the technology is solid. The discussion touched also upon some allergic reactions that have been reported in the media, and that might go.nature.com/37Kf26U). In particular we'd like to explore the gap between academic research and industrial requirements, which is one of aspects of nanotechnology that is seldom discussed in scientific journals, including ours.

Beyond Q\&As and panel discussions, in our 'meet the editors' sessions we shall describe the processes we use to select the papers that we publish and we shall answer any question that our audience may have about the journal. The first such session was on 1 March 2021 (https://go.nature. com/37YQpTO). We shall also host a series of one-to-one conversations with some of our authors to discuss their results in an informal and understandable fashion. For example, the first of such events, which is planned for 24 March, will focus on some of the topics covered in the March issue (https://go.nature.com/2MBSkX2).

We are not unique in organizing webinars during the pandemic and we also appreciate that online conversations cannot replace face-to-face interactions. We believe, however, that a series such as Nature Nano Talks offers the unique advantage of simultaneously reaching a large number of people spread around the globe, which would be impossible to do otherwise. For this reason, we are committed to continue even when travelling to conferences becomes easier for us. We hope you welcome the idea and invite you to regularly check the list of planned events (https://www. nature.com/nnano/video) and register for as many as you wish.

Published online: 4 March 2021 https://doi.org/10.1038/s41565-021-00880-w 